\title{
A stochastic analogue of Aubry-Mather theory*
}

\author{
Diogo Aguiar Gomes \\ Department of Mathematics, University of Texas at Austin, RLM 8.100, 26th and Speedway, \\ Austin, TX 78712-1082, USA \\ and \\ Instituto Superior Tecnico, Dep. de Matematica, Av. Rovisco Pais, 1049-001, Lisbon, Portugal \\ E-mail: dgomes@math.ist.utl.pt
}

Received 26 June 2001, in final form 25 January 2002

Published 11 March 2002

Online at stacks.iop.org/Non/15/581

Recommended by A Chenciner

\begin{abstract}
In this paper, we discuss a stochastic analogue of Aubry-Mather theory in which a deterministic control problem is replaced by a controlled diffusion. We prove the existence of a minimizing measure (Mather measure) and discuss its main properties using viscosity solutions of Hamilton-Jacobi equations. Then we prove regularity estimates on viscosity solutions of the Hamilton-Jacobi equation using the Mather measure. Finally, we apply these results to prove asymptotic estimates on the trajectories of controlled diffusions and study the convergence of Mather measures as the rate of diffusion vanishes.
\end{abstract}

Mathematics Subject Classification: 35J60, 37J40, 49L25, 60H30

\section{Introduction}

The objective of this paper is to understand a stochastic analogue of Aubry-Mather theory. The original problem [Mat91] consists in determining a probability measure $\mu(x, v)$ in $\mathbb{T}^{n} \times \mathbb{R}^{n}$ ( $\mathbb{T}^{n}$ is the $n$-dimensional torus) that minimizes the average action

$$
\int L(x, v) \mathrm{d} \mu
$$

for a given Lagrangian $L$ with the constraint that $\mu$ is invariant under the flow generated by the Euler-Lagrange equations associated with $L$. This problem is equivalent [Mat01] to the relaxed problem of minimizing (1) with the constraint

$$
\int v D_{x} \phi \mathrm{d} \mu=0
$$

\footnotetext{
* Supported in part by FCT (Portugal) through programs POCTI, POCTI/32931/MAT/2000, BPD 1531/2000 and NSF Grant DMS 97-29992. 
for any $\phi(x)$. In the case of controlled diffusions, we replace this constraint by

$$
\int A^{v} \phi \mathrm{d} \mu=0
$$

for all $\phi$ smooth and periodic, in which $A^{v} \phi=v D_{x} \phi+\left(\sigma^{2} / 2\right) \Delta \phi$ is the infinitesimal generator of the controlled diffusion [FS93].

We proceed as follows. In section 2 we construct a relaxed minimization problem on a space of measures. Then, in section 3, we identify its dual by means of the FenchelRockafellar duality theorem [Roc66]. This dual problem turns out to involve Hamilton-Jacobi equations which are studied in section 4 . We prove equivalence between the strong and weak problems (section 5), and characterize the minimizing measures using viscosity solutions of Hamilton-Jacobi equations (section 6). Then we discuss several applications: regularity of Hamilton-Jacobi equations (section 7), logarithmic transform, connection with eigenvalue problems (section 8), asymptotics for controlled diffusions (section 9) and convergence of the stochastic Mather measure as the diffusion coefficient vanishes (section 10).

The original Mather problem as well as its stochastic version are convex linear programming problems over a space of Radon measures. Related control problems have been studied by duality [VL78a, VL78b, LV80, FV89, FV88, Fle89], in which the FenchelRockafellar duality theorem [Roc66] is used to analyse optimal control problems. In this paper, we apply similar techniques to understand Aubry-Mather theory and its stochastic analogues.

Several authors have studied the relation between viscosity solutions of HamiltonJacobi equations and Mather measures [Fat97a, Fat97b, Fat98a, Fat98b, E99, EG01, Gom00a, Gom00b]. The results by Fathi [Fat97a, Fat97b, Fat98a, Fat98b] and Weinan [E99] make clear the connection between viscosity solutions and Hamiltonian dynamics. The main idea is that if, for each $P \in \mathbb{R}^{n}, u(x, P)$ is a viscosity solution of

$$
H\left(x, P+D_{x} u\right)=\bar{H}
$$

for some $\bar{H}(P) \in \mathbb{R}$ (here $H$ is the Legendre transform of $L$ ), then there exists an invariant set $\mathcal{I}$ contained in the graph

$$
\left\{\left(x, P+D_{x} u(x, P)\right)\right\} .
$$

Furthermore, $\mathcal{I}$ is a subset of a Lipschitz graph, i.e. $D_{x} u(x, P)$ is a Lipschitz function on $\pi(\mathcal{I})$, where $\pi(x, p)=x$. If $\bar{H}(P)$ is differentiable at $P$, then any solution $(x(t), p(t))$ of the Hamiltonian dynamics

$$
\dot{x}=-D_{p} H(p, x), \quad \dot{p}=D_{x} H(p, x)
$$

with initial conditions on $\mathcal{I}$ satisfies

$$
\lim _{t \rightarrow \infty} \frac{\left|x(t)+D_{P} \bar{H} t\right|}{t}=0 .
$$

In the previous equation and in the remainder of the paper, we use the convention that trajectories $x(t) \in \mathbb{T}^{n}$ are lifted to $\mathbb{R}^{n}$ whenever it is convenient.

In [Gom00a, Gom00b], this problem is studied in detail and more precise asymptotic results are presented. We also prove regularity results for the viscosity solution of (4) - in particular, under unique ergodicity of the Mather measure, uniform continuity in $P$ (after adding to $u$ a suitable function of $P$ ). In [EG01] Mather measures are used to prove regularity for solutions of Hamilton-Jacobi equations. The main results are $L^{2}$-type estimates in the difference quotients of $D_{x} u$. The objective of this paper is to generalize these results to the stochastic case. 
Most of the results in this paper hold (with appropriate modifications) for compact manifolds - in the classical case this was studied by Fathi in [Fat97a, Fat97b, Fat98a, Fat98b]. In this more general case, $\mathbb{T}^{n} \times \mathbb{R}^{n}$ would be replaced by the tangent space $T M$ of a compact manifold $M$, and $P$ by a cohomology class in $H^{1}(M, \mathbb{R})$. Also we could have used a more general elliptic operator corresponding to the Laplacian.

\section{Stochastic Mather measures}

In this section we define a stochastic analogue of Mather's minimal measure problem [Mat89, Mat91, Mn92, Mn96]. To do so, we consider an ergodic diffusion control problem and study an associated relaxed minimization problem on a space of measures. In the next section, we identify its dual by means of the Fenchel-Rockafellar duality theorem and show that the dual problem is, in some sense, a Hamilton-Jacobi equation.

Consider a controlled Markov diffusion [FS93] in $\mathbb{R}^{n}$,

$$
\mathrm{d} x=\vartheta(t) \mathrm{d} t+\sigma \mathrm{d} w,
$$

where $w$ is an $n$-dimensional Brownian motion, $\vartheta(t)$ is a bounded progressively measurable control, i.e. $\vartheta(t)$ is measurable with respect to the $\sigma$-field generated by $w_{s}, s \leqslant t$ (see [FS93] for more details), and $\sigma \geqslant 0$ the diffusion rate ( $\sigma=0$ corresponds to the standard Aubry-Mather theory). The control objective is to minimize the long-time running cost

$$
\lim _{T \rightarrow+\infty} \frac{1}{T} E \int_{0}^{T} L(x(t), \vartheta(t)) \mathrm{d} t,
$$

over all bounded progressively measurable control processes $\vartheta(t)$; this is called the ergodic control problem (here $E$ denotes the expected value with respect to the underlying probability measure). We assume that the function $L(x, v)$ is smooth in both variables, $\mathbb{Z}^{n}$ periodic in $x$, coercive $\lim _{|v| \rightarrow+\infty}(L(x, v) /|v|)=+\infty$ and strictly convex in $v$, i.e. $D_{v v}^{2} L(x, v) \geqslant \gamma>0$ for some fixed number $\gamma>0$. Furthermore, since adding a constant to $L$ does not change the nature of the problem, we also assume $L \geqslant 0$.

Let $\Omega=\mathbb{T}^{n} \times \mathbb{R}^{n}$, where $\mathbb{T}^{n}$ is the $n$-dimensional torus, identified, when convenient, with $[0,1]^{n}$ or $\mathbb{R}^{n}$ with a periodic structure (in geometric terms, $\mathbb{R}^{n}$ is the universal covering of $\left.\mathbb{T}^{n}\right)$. A pair $(x, v)=z$ represents a generic point $z \in \Omega$, with $x \in \mathbb{T}^{n}$ and $v \in \mathbb{R}^{n}$. Choose a function $\gamma \equiv \gamma(|v|): \Omega \rightarrow[1,+\infty)$ satisfying

$$
\lim _{|v| \rightarrow+\infty} \frac{L(x, v)}{\gamma(v)}=+\infty, \quad \lim _{|v| \rightarrow+\infty} \frac{|v|}{\gamma(v)}=0 .
$$

Let $\mathcal{M}$ be the set of weighted Radon measures on $\Omega$, i.e.

$$
\mathcal{M}=\left\{\text { signed measures on } \Omega \text { with } \int_{\Omega} \gamma \mathrm{d}|\mu|<\infty\right\} .
$$

Note that $\mathcal{M}$ is the dual of the set $C_{\gamma}^{0}(\Omega)$ of continuous functions $\phi$ with

$$
\|\phi\|_{\gamma}=\sup _{\Omega}\left|\frac{\phi}{\gamma}\right|<\infty, \quad \lim _{|z| \rightarrow \infty} \frac{\phi(z)}{\gamma(v)} \rightarrow 0 .
$$

Note that, for any $\varphi \in C^{2}\left(\mathbb{T}^{n}\right), A^{v} \varphi \in C_{\gamma}^{0}(\Omega)$ and so (3) is well defined for $\mu \in \mathcal{M}$.

For each bounded progressively measurable control strategy $\vartheta$, consider the probability measure $\mu_{T}^{\vartheta}$ defined by

$$
\int_{\Omega} \phi \mathrm{d} \mu_{T}^{\vartheta}=\frac{1}{T} E \int_{0}^{T} \phi(x(t), \vartheta(t)) \mathrm{d} t .
$$


Since each $\mu_{T}$ is a probability measure, we may extract a weakly convergent subsequence such that, as $T \rightarrow+\infty, \mu_{T}^{\vartheta} \rightarrow \mu^{\vartheta}$, i.e., for any $\phi \in C_{\gamma}^{0}(\Omega)$,

$$
\int_{\Omega} \phi \mathrm{d} \mu_{T}^{\vartheta} \rightarrow \int_{\Omega} \phi \mathrm{d} \mu^{\vartheta}
$$

Let

$$
\mathcal{M}_{0}=\operatorname{cl}\left\{\mu^{\vartheta}: \vartheta(t) \text { bounded progressively measurable control }\right\}
$$

Define

$$
\mathcal{M}_{1}=\left\{\mu \in \mathcal{M}: \int_{\Omega} \mathrm{d} \mu=1, \mu \geqslant 0\right\} .
$$

The stochastic analogue of Mather's problem consists in determining a measure $\mu$ that minimizes

$$
\inf _{\mu \in \mathcal{M}_{0} \cap \mathcal{M}_{1}} \int_{\Omega} L \mathrm{~d} \mu \text {. }
$$

For our purposes, however, it is convenient to consider a relaxed problem by replacing $\mathcal{M}_{0}$ by a slightly larger set $\mathcal{N}_{0}$ that we define next.

The infinitesimal generator corresponding to the controlled diffusion (7) is

$$
A^{v} \phi=\frac{\sigma^{2}}{2} \Delta \phi+v \cdot \nabla \phi
$$

Proposition 1. Any measure $\mu \in \mathcal{M}_{0}$ satisfies

$$
\int_{\Omega} A^{v} \varphi \mathrm{d} \mu=0
$$

for all $\varphi=\varphi(x), \varphi$ periodic and $C^{2}\left(\right.$ or $C^{1}$ if $\left.\sigma=0\right)$.

Proof. Given a bounded progressively measurable control $\vartheta(t)$, consider the measures $\mu_{T}^{\vartheta}$ and $\mu^{\vartheta}$ as defined, respectively, by (8) and (9). We claim that

$$
\int_{\Omega} A^{v} \varphi \mathrm{d} \mu^{\vartheta}=0
$$

for $\varphi(x) \in C^{2}\left(\mathbb{T}^{n}\right)$. To see this, recall Dynkin's formula (see [FS93, p 391]):

$$
\varphi(x(T))-\varphi(x(0))=E \int_{0}^{T} A^{\vartheta(t)} \varphi(x(t)) \mathrm{d} t
$$

for any $x(t)$ solving (7) with $\vartheta(t)$ bounded progressively measurable. In the case $\sigma=0$, this is just the fundamental theorem of calculus. Dividing by $T$ and letting $T \rightarrow \infty$, we obtain (11).

Let $\mathcal{N}_{0}$ be the closure of the set of all measures that satisfy (11):

$$
\mathcal{N}_{0}=\mathrm{cl}\left\{\mu \in \mathcal{M}: \int_{\Omega} A^{v} \varphi \mathrm{d} \mu=0, \forall \varphi(x) \in C^{2}\left(\mathbb{T}^{n}\right)\right\} .
$$

In the case $\sigma=0$, the set $\mathcal{N}_{0}$ is the 'measure theoretic' analogue of the set of closed curves on $\mathbb{T}^{n}$. Indeed, if $\theta:[0,1] \rightarrow \mathbb{T}^{n}$ is a piecewise smooth closed curve, then we can define a measure $\mu_{\theta}$ by

$$
\int_{\Omega} f \mathrm{~d} \mu_{\theta}=\int_{0}^{1} f(\theta(t), \dot{\theta}(t)) \mathrm{d} t .
$$

Clearly $\mu_{\theta}$ is in $\mathcal{N}_{0}$, and since $\mathcal{N}_{0}$ is a linear space, it contains all linear combinations of measures of this form. If a measure $\mu \in \mathbb{N}_{0}$ is supported on a graph $(x, v(x))$, then the 
condition $\int_{\Omega} A^{v} \varphi \mathrm{d} \mu=0$ for all $\varphi(x)$ implies that $\mu$ is invariant under the semigroup with infinitesimal generator $A^{v(x)}$.

The additional problem that we will consider is

$$
\inf _{\mu \in \mathcal{N}_{0} \cap \mathcal{M}_{1}} \int L \mathrm{~d} \mu \text {. }
$$

We will prove later on that

$$
\inf _{\mu \in \mathcal{N}_{0} \cap \mathcal{M}_{1}} \int L \mathrm{~d} \mu=\inf _{\mu \in \mathcal{M}_{0} \cap \mathcal{M}_{1}} \int L \mathrm{~d} \mu .
$$

This identity is a consequence of $\mathcal{N}_{0}$ being the weak-* closure of $\mathcal{M}_{0}$. However, the proof of this depends on (13) holding for a sufficiently large class of $L$ (see [FV89, FV88, Fle89] for related proofs). Therefore, we will prove (13) directly.

The last issue we discuss in this section is the existence of a measure that minimizes

$$
\inf _{\mu \in \mathcal{M}_{0} \cap \mathcal{M}_{1}} \int L \mathrm{~d} \mu \text {. }
$$

This measure is the stochastic analogue of the Aubry-Mather measure. A similar proof also shows that there exists a minimizing measure in $\mathcal{N}_{0} \cap \mathcal{M}_{1}$. In the next section, we prove that

$$
\inf _{\mu \in \mathcal{M}_{0} \cap \mathcal{M}_{1}} \int L \mathrm{~d} \mu=\inf _{\mu \in \mathcal{N}_{0} \cap \mathcal{M}_{1}} \int L \mathrm{~d} \mu .
$$

First we quote a compacity lemma.

Lemma 1 (Mañé [Mn96]). In $\mathcal{M}_{0} \cap \mathcal{M}_{1}$ the set

$$
\int L \mathrm{~d} \mu<c
$$

is compact with respect to the weak-* topology in $\left(C_{\gamma}^{0}\right)^{\prime}$.

With the help of this lemma, we prove the existence of a minimizing measure.

Theorem 1. There exists a measure $\mu \in \mathcal{M}_{0} \cap \mathcal{M}_{1}$ such that

$$
\int L \mathrm{~d} \mu=\inf _{\mu \in \mathcal{M}_{0} \cap \mathcal{M}_{1}} \int L \mathrm{~d} \mu \text {. }
$$

Proof. Take any minimizing sequence $\mu_{n}$. Since $\int L \mathrm{~d} \mu<c$, the previous lemma shows that by extracting a subsequence, if necessary, $\mu_{n} \stackrel{*}{\rightarrow} \mu$. Thus, for any fixed $k$,

$$
\lim _{n \rightarrow+\infty} \int \min (L, k) \mathrm{d} \mu_{n} \rightarrow \int \min (L, k) \mathrm{d} \mu .
$$

Thus,

$$
\int \min (L, k) \mathrm{d} \mu \leqslant \inf _{\mu \in \mathcal{M}_{0} \cap \mathcal{M}_{1}} \int L \mathrm{~d} \mu
$$

for all $k$. But then by the monotone convergence theorem,

$$
\int L \mathrm{~d} \mu \leqslant \inf _{\mu \in \mathcal{M}_{0} \cap \mathcal{M}_{1}} \int L \mathrm{~d} \mu
$$

which proves the theorem.

A similar proof yields the following theorem.

Theorem 2. There exists a measure $\mu \in \mathcal{N}_{0} \cap \mathcal{M}_{1}$ such that

$$
\int L \mathrm{~d} \mu=\inf _{\mu \in \mathcal{N}_{0} \cap \mathcal{M}_{1}} \int L \mathrm{~d} \mu \text {. }
$$




\section{Identification of the dual problem}

In this section we identify the dual problem of

$$
\min _{\mu \in \mathcal{N}_{0} \cap \mathcal{M}_{1}} \int L \mathrm{~d} \mu \text {. }
$$

The dual problem involves a Hamilton-Jacobi equation. Further analysis of this equation is carried out in the remaining sections and yields important information about the minimizing measure.

First we review some facts about convex duality. Let $E$ be a Banach space with dual $E^{\prime}$. The pairing between $E$ and $E^{\prime}$ is denoted by $(\cdot, \cdot)$. Suppose $h_{1}: E \rightarrow(-\infty,+\infty]$ is a convex, lower semicontinuous function. The Legendre-Fenchel transform $h_{1}^{*}: E^{\prime} \rightarrow[-\infty,+\infty]$ of $h_{1}$ is defined by

$$
h_{1}^{*}(y)=\sup _{x \in E}\left(-(x, y)-h_{1}(x)\right)
$$

for $y \in E^{\prime}$. Similarly, for concave, upper semicontinuous functions $h_{2}: E \rightarrow(-\infty,+\infty]$, let

$$
h_{2}^{*}(y)=\inf _{x \in E}\left(-(x, y)-h_{2}(x)\right) .
$$

Theorem 3 (Rockafellar [Roc66]). Let E be a locally convex Hausdorff topological vector space over $\mathbb{R}$ with dual $E^{*}$. Suppose $h_{1}: E \rightarrow(-\infty,+\infty]$ is convex and lower semicontinuous and $h_{2}: E \rightarrow[-\infty,+\infty)$ is concave and upper semicontinuous. Then

$$
\sup _{x} h_{2}(x)-h_{1}(x)=\inf _{y} h_{1}^{*}(y)-h_{2}^{*}(y),
$$

provided that either $h_{1}$ or $h_{2}$ is continuous at some point where both functions are finite.

For $\phi(x, v) \in C_{\gamma}^{0}(\Omega)$, define

$$
h_{1}(\phi)=\sup _{(x, v) \in \Omega}(-\phi(x, v)-L(x, v)) .
$$

Let $\mathcal{C}$ be defined by

$$
\mathcal{C}=\operatorname{cl}\left\{\phi: \phi(x, v)=A^{v} \varphi(x), \varphi(x) \in C^{2}\left(\mathbb{T}^{n}\right)\right\} .
$$

Here cl denotes the closure in $C_{\gamma}^{0}$ (if $\sigma=0$, we may take $\varphi(x) \in C^{1}\left(\mathbb{T}^{n}\right)$ ). If $\sigma=0$, we may think of the elements in $\mathcal{C}$, as generalized exact differential forms; indeed if $\theta:[0,1] \rightarrow \mathbb{T}^{n}$ is a piecewise smooth closed curve and $\phi \in \mathcal{C}$, then

$$
\int \phi \mathrm{d} \mu_{\theta}=0
$$

Define

$$
h_{2}(\phi)= \begin{cases}0 & \text { if } \phi \in \mathcal{C}, \\ -\infty & \text { otherwise }\end{cases}
$$

In this section we prove that

$$
\sup _{\phi \in C_{\gamma}^{0}(\Omega)} h_{2}(\phi)-h_{1}(\phi)
$$

is the dual problem of (12).

First we compute the Legendre-Fenchel transforms of $h_{1}$ and $h_{2}$ in order to apply theorem 3 to (15). 
Proposition 2. We have

$$
h_{1}^{*}(\mu)= \begin{cases}\int L \mathrm{~d} \mu & \text { if } \mu \in \mathcal{M}_{1}, \\ +\infty & \text { otherwise }\end{cases}
$$

and

$$
h_{2}^{*}(\mu)= \begin{cases}0 & \text { if } \mu \in \mathcal{N}_{0}, \\ -\infty & \text { otherwise. }\end{cases}
$$

Proof. Recall that

$$
h_{1}^{*}(\mu)=\sup _{\phi \in C_{\gamma}^{0}(\Omega)}\left(-\int \phi \mathrm{d} \mu-h_{1}(\phi)\right) .
$$

We claim that if $\mu$ is not positive, then $h_{1}^{*}(\mu)=\infty$.

Lemma 2. If $\mu \ngtr 0$, then $h_{1}^{*}(\mu)=+\infty$.

Proof. If $\mu \ngtr 0$, we can choose a sequence of positive functions $\phi_{n} \in C_{\gamma}^{0}(\Omega)$ such that

$$
\int-\phi_{n} \mathrm{~d} \mu \rightarrow+\infty
$$

Thus, since $L \geqslant 0$,

$$
\sup _{\Omega}-\phi_{n}-L \leqslant 0 .
$$

Therefore if $\mu \ngtr 0$, then $h_{1}^{*}(\mu)=+\infty$.

Lemma 3. If $\mu \geqslant 0$, then

$$
h_{1}^{*}(\mu) \geqslant \int L \mathrm{~d} \mu+\sup _{\psi \in C_{\gamma}^{0}(\Omega)}\left(\int \psi \mathrm{d} \mu-\sup \psi\right) .
$$

Proof. Let $L_{n}$ be a sequence of functions in $C_{\gamma}^{0}(\Omega)$ increasing pointwise to $L$. Any function $\phi$ in $C_{\gamma}^{0}(\Omega)$ can be written as $\phi=-L_{n}-\psi$ for some $\psi$ also in $C_{\gamma}^{0}(\Omega)$. Thus,

$\sup _{\phi \in C_{\gamma}^{0}(\Omega)}\left(-\int \phi \mathrm{d} \mu-h_{1}(\phi)\right)=\sup _{\psi \in C_{\gamma}^{0}(\Omega)}\left(\int L_{n} \mathrm{~d} \mu+\int \psi \mathrm{d} \mu-\sup \left(L_{n}+\psi-L\right)\right)$.

Since $L_{n}-L \leqslant 0$,

$$
\sup _{\Omega} L_{n}-L \leqslant 0
$$

thus

$$
\sup _{\Omega}\left(L_{n}+\psi-L\right) \leqslant \sup _{\Omega} \psi
$$

Thus

$$
\sup _{\phi \in C_{\gamma}^{0}(\Omega)}\left(-\int \phi \mathrm{d} \mu-h_{1}(\phi)\right) \geqslant \sup _{\psi \in C_{\gamma}^{0}(\Omega)}\left(\int L_{n} \mathrm{~d} \mu+\int \psi \mathrm{d} \mu-\sup (\psi)\right) .
$$

By the monotone convergence theorem, $\int L_{n} \mathrm{~d} \mu \rightarrow \int L \mathrm{~d} \mu$. Therefore

$$
\sup _{\phi \in C_{\gamma}^{0}(\Omega)}\left(-\int \phi \mathrm{d} \mu-h_{1}(\phi)\right) \geqslant \int L \mathrm{~d} \mu+\sup _{\psi \in C_{\gamma}^{0}(\Omega)}\left(\int \psi \mathrm{d} \mu-\sup (\psi)\right),
$$

as required. 
If $\int L \mathrm{~d} \mu=+\infty$, then $h_{1}^{*}(\mu)=+\infty$. If $\int \mathrm{d} \mu \neq 1$, then

$$
\sup _{\psi \in C_{\gamma}^{0}(\Omega)}\left(\int \psi \mathrm{d} \mu-\sup \psi\right) \geqslant \sup _{\alpha \in \mathbb{R}} \alpha\left(\int \mathrm{d} \mu-1\right)=+\infty
$$

by taking $\psi \equiv \alpha$, constant. Therefore $h_{1}^{*}(\mu)=+\infty$.

If $\int \mathrm{d} \mu=1$ we have, from the previous lemma,

$$
h_{1}^{*}(\mu) \geqslant \int L \mathrm{~d} \mu
$$

by taking $\psi \equiv 0$.

Also, for any $\phi$,

$$
\int(-\phi-L) \mathrm{d} \mu \leqslant \sup _{\Omega}(-\phi-L)
$$

if $\int \mathrm{d} \mu=1$. Hence

$$
\sup _{\phi \in C_{\gamma}^{0}(\Omega)}\left(-\int \phi \mathrm{d} \mu-h_{1}(\phi)\right) \leqslant \int L \mathrm{~d} \mu .
$$

Thus

$$
h_{1}^{*}(\mu)= \begin{cases}\int L \mathrm{~d} \mu & \text { if } \mu \in \mathcal{M}_{1}, \\ +\infty & \text { otherwise. }\end{cases}
$$

Now we will compute $h_{2}^{*}$. First observe that if $\mu \notin \mathcal{N}_{0}$, then there exists $\hat{\phi} \in \mathcal{C}$ such that

$$
\int \hat{\phi} \mathrm{d} \mu \neq 0
$$

and so

$$
\inf _{\phi \in \mathcal{C}}-\int \phi \mathrm{d} \mu \leqslant \inf _{\alpha \in \mathbb{R}} \alpha \int \hat{\phi} \mathrm{d} \mu=-\infty .
$$

If $\mu \in \mathcal{N}_{0}$, then $\int \phi \mathrm{d} \mu=0$ for all $\phi \in \mathcal{C}$. Therefore

$$
h_{2}^{*}(\mu)=\inf _{\phi \in \mathcal{C}}-\int \phi \mathrm{d} \mu= \begin{cases}0 & \text { if } \mu \in \mathcal{N}_{0}, \\ -\infty & \text { otherwise. }\end{cases}
$$

This completes the proof of proposition 2 .

The Fenchel-Rockafellar duality theorem states that

$$
\sup _{\phi \in C_{\gamma}^{0}(\Omega)}\left(h_{2}(\phi)-h_{1}(\phi)\right)=\inf _{\mu \in \mathcal{M}}\left(h_{1}^{*}(\mu)-h_{2}^{*}(\mu)\right),
$$

provided on the set $h_{2}>-\infty, h_{1}$ is continuous. In the next lemma we prove that $h_{1}$ is continuous, and therefore (16) holds.

Lemma 4. $h_{1}$ is continuous.

Proof. Suppose $\phi_{n} \rightarrow \phi$ in $C_{\gamma}^{0}$. We must prove that $h_{1}\left(\phi_{n}\right) \rightarrow h_{1}(\phi)$. Observe that $\left\|\phi_{n}\right\|_{\gamma}$ and $\|\phi\|_{\gamma}$ are bounded uniformly by some constant $C$. The growth condition on $L$ implies that there exists $R>0$ such that

$$
\sup _{\Omega}-\hat{\phi}-L=\sup _{\mathbb{T}^{n} \times B_{R}}-\hat{\phi}-L
$$

for all $\hat{\phi}$ in $C_{\gamma}^{0}(\Omega)$ with $\|\hat{\phi}\|_{\gamma}<C$, in which $B_{R}=\left\{v \in \mathbb{R}^{n}:|v| \leqslant R\right\}$ is the ball of radius $R$ centred at the origin. On $B_{R}, \phi_{n} \rightarrow \phi$ uniformly and so

$$
\sup _{\Omega}-\phi_{n}-L \rightarrow \sup _{\Omega}-\phi-L \text {. }
$$


Denote by $H^{\star}$ the value

$$
H^{\star}=-\sup _{\phi \in C_{\gamma}^{0}(\Omega)}\left(h_{2}(\phi)-h_{1}(\phi)\right) .
$$

Theorem 4. We have

$$
H^{\star}=\inf \left\{\lambda: \exists \varphi \in C^{2}\left(\mathbb{T}^{n}\right):-\frac{\sigma^{2}}{2} \Delta \varphi+H\left(x, D_{x} \varphi\right)<\lambda\right\},
$$

in which

$$
H(x, p)=\sup _{v}-p \cdot v-L(x, v)
$$

is the Legendre transform of $L$.

Proof. Note that

$H^{\star}=\inf _{\varphi \in C^{2}\left(T^{n}\right)} \sup _{(x, v) \in \Omega}-\frac{\sigma^{2}}{2} \Delta \varphi-v D_{x} \varphi-L=\inf _{\varphi \in C^{2}\left(\mathbb{T}^{n}\right)} \sup _{x \in \mathbb{T}^{n}}-\frac{\sigma^{2}}{2} \Delta \varphi+H\left(x, D_{x} \varphi\right)$.

We should remark that this formula for $H^{\star}$ (which turns out to be equal to $\bar{H}$; see the next section) was proved, in the deterministic case, in [CIPP98].

\section{The cell problem}

The last theorem in the previous section suggests that we study the equation

$$
-\frac{\sigma^{2}}{2} \Delta u+H\left(x, D_{x} u\right)=\bar{H} .
$$

In this section, we prove that there exists a unique number $\bar{H}$ for which (17) has a periodic viscosity solution. Using the results from [Kry87], we show that such a solution is $C^{2}$. Then we prove that the solution is unique (up to additive constants). Finally we prove estimates on $\bar{H}$ and $u$ that do not depend on $\sigma$.

Theorem 5. For $\sigma>0$, there exists a unique number $\bar{H}$ for which the equation

$$
-\frac{\sigma^{2}}{2} \Delta u+H\left(x, D_{x} u\right)=\bar{H}
$$

has a periodic viscosity solution. Furthermore, the solution is $C^{2}$ and unique up to constants.

Remark. When compared to the case $\sigma=0$, this result is extremely interesting because, although $\bar{H}$ is still unique, the viscosity solution of $H\left(D_{x} u, x\right)=\bar{H}$ is not unique up to constants.

Proof. First we address the issue of the existence of a viscosity solution. To do so, consider the infinite horizon discounted cost problem

$$
u^{\alpha}=\inf E \int_{0}^{\infty} \mathrm{e}^{-\alpha t} L(x, \vartheta) \mathrm{d} t
$$

with $\mathrm{d} x=\vartheta \mathrm{d} t+\sigma \mathrm{d} w$, and the infimum is taken over bounded progressively measurable controls $\vartheta(t)$. We consider this problem in the limit $\alpha \rightarrow 0$. Then $u^{\alpha}$ is a periodic viscosity solution of [FS93]

$$
-\frac{\sigma^{2}}{2} \Delta u^{\alpha}+H\left(x, D_{x} u^{\alpha}\right)+\alpha u^{\alpha}=0 .
$$


Since $u^{\alpha}$ is periodic and uniformly Lipschitz in $\alpha$ [FS93], there exists a subsequence $u^{\alpha}$ and $u$ periodic for which

$$
u^{\alpha}-\min u^{\alpha} \rightarrow u
$$

as $\alpha \rightarrow 0$. The zero control $\vartheta \equiv 0$ is bounded and progressively measurable; thus we have the estimate

$$
u^{\alpha} \leqslant \int_{0}^{\infty} \mathrm{e}^{-\alpha t} L(x, 0) \mathrm{d} t \leqslant \frac{C}{\alpha} .
$$

Also because $L \geqslant 0$, we have $u^{\alpha} \geqslant 0$. Thus $0 \leqslant u^{\alpha} \leqslant C / \alpha$, and so we have $\alpha u^{\alpha} \rightarrow-\bar{H}$ for some $\bar{H}$ (extracting a further subsequence if necessary). Then $u$ is a periodic viscosity solution of

$$
-\frac{\sigma^{2}}{2} \Delta u+H\left(x, D_{x} u\right)=\bar{H} .
$$

This solution $u$ is actually $C^{2}$ by standard regularity results for non-linear uniformly elliptic equations [Kry87].

To prove uniqueness of $\bar{H}$, suppose, by contradiction, that $u_{i}$ and $\bar{H}_{i}(i=1,2)$ solve

$$
-\Delta u_{i}+H\left(x, D_{x} u_{i}\right)=\bar{H}_{i} .
$$

Suppose $u_{1}-u_{2}$ has a local maximum at $x_{0}$. Then $D_{x} u_{1}=D_{x} u_{2}$ and $\Delta u_{1} \leqslant \Delta u_{2}$ at $x_{0}$. Thus we conclude $\bar{H}_{1} \geqslant \bar{H}_{2}$. By symmetry, $\bar{H}_{1}=\bar{H}_{2}$.

The uniqueness of the viscosity solution is proved in the following lemma.

Lemma 5. There exists a unique (up to constants) viscosity solution of

$$
-\Delta u+H\left(x, D_{x} u\right)=\bar{H} \text {. }
$$

Proof. To prove that the viscosity solution is unique, suppose, by contradiction, that $u$ and $v$ are two distinct solutions (i.e. $u-v$ is non-constant) of

$$
-\Delta u+H\left(x, D_{x} u\right)=\bar{H} .
$$

Without loss of generality, we may assume that some small ball centred at the origin of radius $\gamma$ does not contain any critical point of $x_{0}$ of $u(x)-v(x)$ (otherwise, for convenience, we may shift the coordinates). Fix $\epsilon, \lambda>0$. Note that

$$
u(x)-v(x)-\epsilon \mathrm{e}^{-\lambda|x|^{2}}
$$

has a local minimum at $x_{\epsilon, \lambda}$ with $\left|x_{\epsilon, \lambda}\right| \leqslant C$ independently of $\epsilon$ and $\lambda$. This holds because $u-v$ is periodic and $\mathrm{e}^{-\lambda|x|^{2}}$ decreases as $|x| \rightarrow \infty$; therefore, the global minimum in (18) must occur close to the origin. At $x_{\epsilon, \lambda}$, we have

$$
D_{x} u=D_{x} v-2 \epsilon \lambda x \mathrm{e}^{-\lambda|x|^{2}}
$$

and

$$
\Delta u \geqslant \Delta v-2 \epsilon \lambda n \mathrm{e}^{-\lambda|x|^{2}}+4 \epsilon \lambda^{2}|x|^{2} \mathrm{e}^{-\lambda|x|^{2}}
$$

Since

$$
0=-\Delta(u-v)+H\left(x, D_{x} u\right)-H\left(x, D_{x} v\right),
$$

we have

$$
0 \leqslant 2 \epsilon \lambda n \mathrm{e}^{-\lambda\left|x_{\epsilon, \lambda}\right|^{2}}-4 \epsilon \lambda^{2}\left|x_{\epsilon, \lambda}\right|^{2} \mathrm{e}^{-\lambda\left|x_{\epsilon, \lambda}\right|^{2}}+\mathrm{O}\left(\epsilon \lambda \mathrm{e}^{-\lambda\left|x_{\epsilon, \lambda}\right|^{2}}\right) .
$$

Since there is a neighbourhood of the origin that does not contain a minimizer of $u-v$, for $\epsilon$ small enough $\left|x_{\epsilon, \lambda}\right|>\gamma / 2$. Dividing by $\epsilon \mathrm{e}^{-\lambda\left|x_{\epsilon, \lambda}\right|^{2}}$ and letting $\epsilon \rightarrow 0$, we observe that

$$
\gamma^{2} \lambda^{2}-C \lambda \leqslant 0
$$

Therefore, sending $\lambda \rightarrow \infty$ yields a contradiction.

This yields the desired result. 
Proposition 3. $\bar{H}$ can be estimated independently of $\sigma$ by

$$
\inf _{x} H(x, 0) \leqslant \bar{H} \leqslant \sup _{x} H(x, 0) .
$$

Proof. Suppose $u$ has a minimum at $x_{0}$. Then $-\left(\sigma^{2} / 2\right) \Delta u\left(x_{0}\right) \leqslant 0$ and $D_{x} u\left(x_{0}\right)=0$. Thus,

$$
\bar{H}=-\frac{\sigma^{2}}{2} \Delta u\left(x_{0}\right)+H\left(x_{0}, D_{x} u\right) \leqslant H\left(x_{0}, 0\right) \leqslant \sup _{x} H(x, 0) .
$$

The other estimate is similar.

Finally, we recall that standard estimates for controlled diffusions [FS93] also yield that $u$ is semiconcave (with semiconcavity constant independent of $\sigma$ ) and Lipschitz (also independently of $\sigma$ ).

\section{Equivalence between weak and strong problems}

The next task is to prove that the value $H^{\star}$, computed by considering an infimum over measures in $\mathcal{N}_{0}$, is the same as

$$
\tilde{H}=-\inf _{\mu \in \mathcal{M}_{0}}\left(h_{1}^{*}(\mu)-h_{2}^{*}(\mu)\right)
$$

which in turn coincides with $\bar{H}$ from the previous section. A useful characterization of $\tilde{H}$ is the following theorem.

Theorem 6. $\tilde{H}$ coincides with the unique value $\bar{H}$ for which the equation

$$
-\frac{\sigma^{2}}{2} \Delta u+H\left(D_{x} u, x\right)=\bar{H}
$$

has a periodic viscosity solution.

Proof. We know from theorem 5 that there is a single number $\bar{H}$ for which (19) admits a periodic viscosity solution $u$. We can use that solution to build a Markov feedback strategy to control the diffusion:

$$
\mathrm{d} x=-D_{p} H\left(D_{x} u, x\right) \mathrm{d} t+\sigma \mathrm{d} w .
$$

By Dynkin's formula, a measure $\mu \in \mathcal{M}_{0} \cap \mathcal{M}_{1}$ corresponds to this diffusion for which

$$
\int L \mathrm{~d} \mu=-\bar{H}
$$

Thus

$$
-\bar{H} \geqslant \inf _{\mu \in \mathcal{M}_{0} \cap \mathcal{M}_{1}} \int L \mathrm{~d} \mu=-\tilde{H} .
$$

Now suppose that $-\tilde{H}<-\bar{H}$. This means that there exists $\epsilon>0$, a measure $\mu^{\epsilon} \in \mathcal{M}_{0} \cap \mathcal{M}_{1}$ and a bounded progressively measurable control $\vartheta^{\epsilon}$ such that

$$
\int_{\Omega} L \mathrm{~d} \mu^{\epsilon}=\liminf _{T \rightarrow+\infty} E \frac{1}{T} \int_{0}^{T} L\left(x^{\epsilon}, \vartheta^{\epsilon}\right) \mathrm{d} t<-\bar{H}-\epsilon
$$

Thus

$$
u(x)=\inf _{\vartheta} E \int_{0}^{T} L(x, \vartheta)+\bar{H} \mathrm{~d} t+u(x(T)) \leqslant E \int_{0}^{T} L\left(x^{\epsilon}, \vartheta^{\epsilon}\right)+\bar{H} \mathrm{~d} t+u\left(x^{\epsilon}(T)\right) .
$$

The hypothesis in $\vartheta^{\epsilon}$ implies that there exists a sequence $T_{n} \rightarrow+\infty$ such that

$$
E \int_{0}^{T} L\left(x^{\epsilon}, \vartheta^{\epsilon}\right)+\bar{H} \mathrm{~d} t \leqslant-\frac{\epsilon}{2} T_{n}
$$

which is a contradiction for $T_{n}$ sufficiently large since $u$ is bounded. 
Theorem 7. $H^{\star}$ is the unique value for which the equation

$$
-\frac{\sigma^{2}}{2} \Delta u+H\left(x, D_{x} u\right)=H^{\star}
$$

has a periodic viscosity solution.

Proof. First suppose $u$ is a periodic viscosity solution of

$$
-\frac{\sigma^{2}}{2} \Delta u+H\left(x, D_{x} u\right)=\bar{H} .
$$

Then we claim that there is no smooth function $\psi$ with

$$
-\frac{\sigma^{2}}{2} \Delta \psi+H\left(x, D_{x} \psi\right)<\bar{H} .
$$

Indeed, if this were false, we could choose a point $x_{0}$ at which $u-\psi$ has a local minimum. At this point, we would have

$$
-\frac{\sigma^{2}}{2} \Delta \psi+H\left(x_{0}, D_{x} \psi\right) \geqslant \bar{H}
$$

by the viscosity property. Hence $H^{\star} \geqslant \bar{H}$ by theorem 4 .

To prove the other inequality, consider a standard mollifier $\eta_{\epsilon}$ and define $u_{\epsilon}=\eta_{\epsilon} * u$, in which $*$ denotes convolution. Then

$$
-\frac{\sigma^{2}}{2} \Delta u_{\epsilon}+H\left(x, D_{x} u_{\epsilon}\right) \leqslant \bar{H}+h(\epsilon, x),
$$

where

$$
h(\epsilon, x)=\sup _{|p| \leqslant R} \sup _{|x-y| \leqslant \epsilon}|H(x, p)-H(y, p)|,
$$

where $R$ is a bound on the Lipschitz constant of $u$. Let

$$
H^{\epsilon}=\bar{H}+\sup _{x} h(\epsilon, x) \text {. }
$$

$u_{\epsilon}$ satisfies

$$
-\frac{\sigma^{2}}{2} \Delta u_{\epsilon}+H\left(x, D_{x} u_{\epsilon}\right) \leqslant H^{\epsilon} .
$$

Thus $H^{\star} \leqslant \lim _{\epsilon \rightarrow 0} H^{\epsilon}=\bar{H}$. Hence $H^{\star}=\bar{H}$.

This proof holds even when $\sigma=0$; for $\sigma \neq 0$, since $u$ is $C^{2}$, the mollification step is unnecessary.

Corollary 1. We have

$$
\inf _{\mu \in \mathcal{N}_{0}}\left(h_{1}^{*}(\mu)-h_{2}^{*}(\mu)\right)=\inf _{\mu \in \mathcal{M}_{0}}\left(h_{1}^{*}(\mu)-h_{2}^{*}(\mu)\right) .
$$

Proof. Our previous results show that we can construct a probability measure $\mu$ on $\mathcal{M}_{0}$ such that

$$
\int L \mathrm{~d} \mu=-\bar{H}=\inf _{\mu \in \mathcal{N}_{0}}\left(h_{1}^{*}(\mu)-h_{2}^{*}(\mu)\right) .
$$

Since $\mathcal{M}_{0} \subset \mathcal{N}_{0}$, this completes the proof. 


\section{Properties of stochastic Mather measures}

In this section we study general properties of stochastic Mather measures. First we prove that the stochastic Mather measure is supported in the graph $\left(x,-D_{p} H\left(D_{x} u, x\right)\right)$ for any $u$ viscosity solution of (19). Then we show that the projection of this measure in the $x$-axis has a density that satisfies an elliptic partial differential equation.

Theorem 8. Any stochastic Mather measure is supported in the graph $\left(x,-D_{p} H\left(x, D_{x} u\right)\right)$ for any $u$ viscosity solution of (19).

Proof. Recall that, for any $v$, we have

$$
-\frac{\sigma^{2}}{2} \Delta u-v D_{x} u-L(x, v) \leqslant \bar{H}
$$

with strict inequality unless $v=-D_{p} \bar{H}\left(x, D_{x} u\right)$. Note that

$$
\int-\frac{\sigma^{2}}{2} \Delta u-v D_{x} u \mathrm{~d} \mu=0
$$

and

$$
-\int L(x, v) \mathrm{d} \mu=\bar{H} .
$$

Thus $\mu$ is supported on $\left(x,-D_{p} H\left(x, D_{x} u\right)\right)$; otherwise we would have

$$
-\int L(x, v) \mathrm{d} \mu<\bar{H}
$$

which would be a contradiction.

Since any stochastic Mather measure is supported on a graph, a natural question is whether its projection in the $x$ coordinates has a density. The answer to this question is affirmative, and we prove that this density is the solution of an elliptic partial differential equation. Before stating the result, recall that $W^{1,2}$ is the Sobolev space of weakly differentiable functions $w$ with $\int w^{2}+\left|D_{x} w\right|^{2} \leqslant \infty$.

Theorem 9. Let $\mu$ be a stochastic Mather measure. Let $v$ denote the projection of $\mu$ in the $x$ coordinates. Then $v=\theta(x) \mathrm{d} x$ for some density $\theta \in W^{1,2}$. Furthermore, $\theta$ is a weak solution of

$$
-\nabla \cdot(\theta v(x))+\frac{1}{2} \sigma^{2} \Delta \theta=0
$$

for $v=-D_{p} H\left(x, D_{x} u\right)$.

Proof. Recall that, for any smooth and periodic $\phi(x)$,

$$
\int\left(\frac{\sigma^{2}}{2} \Delta \phi+v(x) D_{x} \phi\right) \mathrm{d} v=0 .
$$

Let $\eta_{\epsilon}$ be a standard mollifier, $\phi_{\epsilon}=\eta_{\epsilon} * \eta_{\epsilon} * v$ and $v_{\epsilon}=\eta_{\epsilon} * v$. Note that $v_{\epsilon}$ is a bounded periodic $C^{\infty}$ function (the bounds may depend on $\epsilon$ ). Then

$$
0=\int \frac{\sigma^{2}}{2}\left|D_{x} \nu_{\epsilon}\right|^{2} \mathrm{~d} x-\int v(x) D_{x}\left(\phi_{\epsilon}\right) \mathrm{d} \nu .
$$

Thus

$$
\int \frac{\sigma^{2}}{2}\left|D_{x} v_{\epsilon}\right|^{2} \mathrm{~d} x=\int\left(D_{x} v_{\epsilon}\right) \eta_{\epsilon} *(v v) \mathrm{d} x
$$


Note that

$\left|\int\left(D_{x} v_{\epsilon}\right) \eta_{\epsilon} *(v v) \mathrm{d} x\right| \leqslant\left|\int\left(D_{x} v_{\epsilon}\right) v(x) v_{\epsilon} \mathrm{d} x\right|+\left|\int\left(D_{x} v_{\epsilon}\right)\left(\eta_{\epsilon} *(v v)-v(x) v_{\epsilon}\right) \mathrm{d} x\right|$.

The first term on the right-hand side can be estimated by

$$
\frac{\gamma}{2} \int\left|D_{x} v_{\epsilon}\right|^{2} \mathrm{~d} x+\frac{C}{\gamma} \int\left|v_{\epsilon}\right|^{2} \mathrm{~d} x
$$

for any small $\gamma>0$. To estimate the second term, observe that, since $v$ is Lipschitz,

$$
\left|\eta_{\epsilon} *(v v)-v(x) v_{\epsilon}\right| \leqslant \int \eta_{\epsilon}(x-y)|v(x)-v(y)| \mathrm{d} v(y) \leqslant C \epsilon v_{\epsilon}(x) .
$$

Thus

$$
\left|\int D_{x} v_{\epsilon}\left(\eta_{\epsilon} *(v v)-v(x) v_{\epsilon}\right) \mathrm{d} x\right| \leqslant \frac{\gamma}{2} \int\left|D_{x} v_{\epsilon}\right|^{2} \mathrm{~d} x+\frac{C \epsilon}{\gamma} \int\left|v_{\epsilon}\right|^{2} \mathrm{~d} x ;
$$

therefore we conclude that

$$
\int\left|D_{x} v_{\epsilon}\right|^{2} \mathrm{~d} x \leqslant C \int\left|v_{\epsilon}\right|^{2} \mathrm{~d} x
$$

uniformly in $\epsilon$. Now observe that $\nu_{\epsilon} \geqslant 0$ and

$$
\int v_{\epsilon} \mathrm{d} x=1
$$

If $\int\left|\nu_{\epsilon}\right|^{2} \mathrm{~d} x$ were unbounded, then we could normalize it defining $\alpha_{\epsilon}=\gamma_{\epsilon} v_{\epsilon}$ with $\int\left|\alpha_{\epsilon}\right|^{2} \mathrm{~d} x=1$ and $\gamma_{\epsilon} \rightarrow 0$. Since $\alpha_{\epsilon} \in W^{1,2}$ uniformly, through some subsequence it converges in $L^{2}$ to some $\alpha \in L^{2}$ with $\int|\alpha|^{2} \mathrm{~d} x=1$. However, $\alpha \geqslant 0$ and $\int \alpha=0$, which is a contradiction. Therefore, we must have $v_{\epsilon} \in W^{1,2}$ uniformly in $\epsilon$. Thus through some subsequence, $v_{\epsilon} \rightarrow \theta$ for some $\theta \in W^{1,2}$. Thus $\mathrm{d} \nu=\theta(x) \mathrm{d} x$. Consequently, $\theta$ is a weak solution of

$$
-\nabla \cdot(\theta v(x))+\frac{1}{2} \sigma^{2} \Delta \theta=0 .
$$

Observe that equation (20) is a non-symmetric zero eigenvalue problem. It is well known [PW84] that

$$
-\nabla \cdot(\theta v(x))+\frac{1}{2} \sigma^{2} \Delta \theta=\lambda \theta
$$

has a principal eigenvalue $\lambda$ with positive eigenfunction $\theta$. To see that $\lambda=0$, just observe that

$$
0=\int \frac{\sigma^{2}}{2} \Delta \theta-\nabla \cdot(v(x) \theta(x))=\lambda \int \theta .
$$

Since $\theta$ is non-negative, we get $\lambda=0$.

Corollary 2. The invariant measure $\theta$ is unique.

Proof. Since $\theta \geqslant 0$ and $\int_{\mathbb{T}^{n}} \theta \mathrm{d} x=1$, there exists a point $x_{0}$ such that $\theta\left(x_{0}\right)>0$. Then Harnack's inequality for elliptic equations implies $\theta>0$ everywhere. Define $w(x)$ by

$$
\theta=\mathrm{e}^{w} \text {. }
$$

It suffices to prove that $w$ is unique. To do so, observe that $w$ satisfies

$$
\frac{\sigma^{2}}{2} \Delta w+\frac{\sigma^{2}\left|D_{x} w\right|^{2}-2 D_{x} w v}{2}-\nabla \cdot v=0 .
$$

Then the uniqueness lemma 5 implies that $w$ is unique up to constants, i.e. $\theta$ is unique up to a normalizing factor. 
The previous theorem yields several important identities that we will use in the next section. First define $\bar{H}(P)$ to be a number for which

$$
-\frac{\sigma^{2}}{2} \Delta u+H\left(x, P+D_{x} u\right)=\bar{H}(P)
$$

has a periodic viscosity solution $u(x, P)$ - because we can add arbitrary functions of $P$ to $u$ and still get a solution of (21), we cannot assume that $u$ is continuous in $P$. The function $\bar{H}(P)$ is convex in $P$ and so twice differentiable for almost every $P$.

Proposition 4. For any $\phi(x)$ periodic,

$-\int D_{x} \phi(x) D_{p} H\left(x, P+D_{x} u\right) \theta(x, P) \mathrm{d} x+\frac{\sigma^{2}}{2} \int \Delta \phi(x) \theta(x, P) \mathrm{d} x=0$.

Furthermore,

$$
\int D_{x} H\left(x, P+D_{x} u\right) \theta(x, P) \mathrm{d} x=0 .
$$

Finally, for any $P$ and $P^{\prime}$,

$$
\left(P^{\prime}-P\right) \int D_{p} H\left(x, P+D_{x} u\right) \theta(x, P) \mathrm{d} x \leqslant \bar{H}\left(P^{\prime}\right)-\bar{H}(P)
$$

in particular if $\bar{H}$ is differentiable,

$$
\int D_{p} H\left(x, P+D_{x} u\right) \theta(x, P) \mathrm{d} x=D_{P} \bar{H}(P) .
$$

Proof. To prove (22) and (23), we will assume, without loss of generality, that $P=0$ and omit the arguments of the function in order to simplify notation. Observe that (22) follows from

$$
0=\int \phi(x)\left(\nabla \cdot\left(\theta D_{p} H\right)+\frac{1}{2} \sigma^{2} \Delta \theta\right) \mathrm{d} x
$$

by integration by parts.

Let $\eta_{\epsilon}$ be a standard mollifier and let $u_{\epsilon}=\eta_{\epsilon} * u$. Then

$$
-\frac{\sigma^{2}}{2} \Delta u_{\epsilon}+\eta_{\epsilon} * H\left(x, D_{x} u\right)=\bar{H}
$$

Differentiate the previous identity with respect to $x_{i}$ :

$$
-\frac{\sigma^{2}}{2} \Delta D_{x_{i}} u_{\epsilon}+\eta_{\epsilon} *\left(H_{p_{j}} D_{x_{j} x_{i}} u+H_{x_{i}}\right)=0 .
$$

Since $H_{p_{j}}$ is Lipschitz in $x$, we have

$$
\eta_{\epsilon} *\left(H_{p_{j}} D_{x_{j} x_{i}} u\right)=H_{p_{j}} D_{x_{j} x_{i}} u_{\epsilon}+\mathrm{O}(\epsilon) .
$$

Note also that

$$
\int\left(-\frac{\sigma^{2}}{2} \Delta D_{x_{i}} u_{\epsilon}+H_{p_{j}} D_{x_{j} x_{i}}^{2} u_{\epsilon}\right) \theta \mathrm{d} x=0
$$

since $D_{x_{i}} u_{\epsilon}$ is smooth and periodic. Thus

$$
\int \eta_{\epsilon} * H_{x_{i}} \theta \mathrm{d} x \rightarrow 0
$$

as $\epsilon \rightarrow 0$. Since $\eta_{\epsilon} * H_{x_{i}} \rightarrow H_{x_{i}}$ almost everywhere, we conclude

$$
\int H_{x_{i}} \theta \mathrm{d} x=0,
$$

which proves (23). 
To prove the last part of the proposition, note that

$$
\begin{aligned}
& H\left(x, P^{\prime}+D_{x} u\left(x, P^{\prime}\right)\right)-H\left(x, P+D_{x} u(x, P)\right) \\
& \geqslant D_{p} H\left(x, P+D_{x} u(x, P)\right)\left[P^{\prime}+D_{x} u\left(x, P^{\prime}\right)-P-D_{x} u(x, P)\right] .
\end{aligned}
$$

Let $w=u\left(x, P^{\prime}\right)-u(x, P)$. Note that

$$
\int\left(-\frac{\sigma^{2}}{2} \Delta w+D_{p} H\left(x, P+D_{x} u(x, P)\right) D_{x} w\right) \theta \mathrm{d} x=0 .
$$

Thus, by integrating (25) with respect to $\theta$ and using the previous identity, we obtain

$$
\left(P^{\prime}-P\right) \int D_{p} H\left(x, P+D_{x} u(x, P)\right) \leqslant \bar{H}\left(P^{\prime}\right)-\bar{H}(P),
$$

as required.

\section{Regularity estimates}

In this section, we prove $L^{2}$-type regularity estimates for the solution of (21). These estimates are expressed using the invariant measures. A major advantage is that it is possible to prove $L^{2}(\theta)$ estimates for the difference quotient $\left|D_{x} u(x+y)-D_{x} u(x)\right|$ that do not depend on $\sigma$ explicitly, whereas pointwise or $L^{2}$ estimates with respect to Lebesgue measure depend on $\sigma$. Therefore, our estimates extend up to the case $\sigma=0$; for a careful study of this case, consult [EG01, Gom00a, Gom00b].

Theorem 10. Suppose u solves (21) and $y \in \mathbb{R}^{n}$. Then

$$
\int\left|D_{x} u(x+y)-D_{x} u(x)\right|^{2} \theta \mathrm{d} x \leqslant C|y|^{2} .
$$

Furthermore, if $\bar{H}(P)$ is twice differentiable at $P$, then

$$
\int\left|D_{x} u(x, P)-D_{x} u\left(x, P^{\prime}\right)\right|^{2} \theta \mathrm{d} x \leqslant C\left|P-P^{\prime}\right|^{2}
$$

for $\left|P-P^{\prime}\right|$ sufficiently small, and the constant $C$ depends only on bounds for $D_{P P}^{2} \bar{H}$.

Proof. Note that

$-\frac{\sigma^{2}}{2}[\Delta u(x+y)-\Delta u(x)]+H\left(x+y, D_{x} u(x+y)\right)-H\left(x, D_{x} u(x)\right)=0$.

Since $H$ is convex,

$$
\begin{aligned}
& H\left(x+y, D_{x} u(x+y)\right)-H\left(x, D_{x} u\right) \\
& \quad \geqslant \gamma\left|D_{x} w\right|^{2}+D_{p} H\left(x, D_{x} u(x)\right) D_{x} w+D_{x} H\left(x, D_{x} u(x)\right) y+\mathrm{O}\left(y^{2}\right),
\end{aligned}
$$

with $w=u(x+y)-u(x)$. Integrating with respect to $\theta \mathrm{d} x$ and observing that $\left\|D_{x} u\right\|_{\infty}$ is bounded independently of $\sigma$, we obtain

$$
\gamma \int\left|D_{x} w\right|^{2} \theta \mathrm{d} x \leqslant C|y|^{2}
$$

since

$$
\int\left[D_{p} H\left(x, D_{x} u(x)\right) D_{x} w-\frac{\sigma^{2}}{2} \Delta w\right] \theta \mathrm{d} x=0
$$

and

$$
\int D_{x} H\left(x, D_{x} u(x)\right) \theta \mathrm{d} x=0 .
$$


Similarly, let $w=u\left(x, P^{\prime}\right)-u(x, P)$ and assume $\bar{H}(P)$ is twice differentiable at $P$. Then

$$
\begin{aligned}
D_{P} \bar{H}(P)\left(P^{\prime}-P\right)+C\left|P-P^{\prime}\right|^{2} & \\
& \geqslant-\frac{\sigma^{2}}{2} \Delta w+H\left(x, P^{\prime}+D_{x} u\left(x, P^{\prime}\right)\right)-H\left(x, P+D_{x} u(x, P)\right) .
\end{aligned}
$$

Note that

$$
\begin{aligned}
& H\left(x, P^{\prime}+D_{x} u\left(x, P^{\prime}\right)\right)-H\left(x, P+D_{x} u(x, P)\right) \\
& \quad \geqslant D_{p} H\left(x, P+D_{x} u(x, P)\right)\left(P^{\prime}-P+D_{x} w\right)+\gamma\left|P^{\prime}-P+D_{x} w\right|^{2} .
\end{aligned}
$$

Thus

$$
\gamma \int\left|P^{\prime}+D_{x} u\left(x, P^{\prime}\right)-P-D_{x} u(x, P)\right|^{2} \theta \mathrm{d} x \leqslant C\left|P-P^{\prime}\right|^{2},
$$

since

$$
\left(P^{\prime}-P\right) \int D_{p} H\left(x, P+D_{x} u(x, P)\right) \theta \mathrm{d} x=\left(P^{\prime}-P\right) D_{P} \bar{H}
$$

and

$$
\int-\frac{\sigma^{2}}{2} \Delta w+D_{p} H\left(x, P+D_{x} u(x, P)\right) D_{x} w=0 .
$$

From (28) we have (27), using $(a+b)^{2} \leqslant C_{1} a^{2}$ implies $b^{2} \leqslant C_{2} a^{2}$ for some constant $C_{2}$.

In the next theorem, we prove that if $\bar{H}$ is strictly convex in a neighbourhood of a point $P$, i.e.

$$
\bar{H}\left(P^{\prime}\right) \geqslant \bar{H}(P)+\omega \cdot\left(P^{\prime}-P\right)+C\left|P^{\prime}-P\right|^{2},
$$

supporting plane $\omega$ of $\bar{H}$ at $P$ and some constant $\gamma>0$, then the map $(x, P) \rightarrow P+D_{x} u(x, P)$ is non-degenerate. In the non-random case, this result is extremely important since it proves that the invariant sets contained in $\left(x, P+D_{x} u\right)$ change with $P$; see [Gom00a] for a detailed discussion.

Theorem 11. Suppose $\bar{H}$ is strictly convex at a neighbourhood of a point $P$ and differentiable at $P$. Then

$$
\int\left|P+D_{x} u(x, P)-P^{\prime}-D_{x} u\left(x, P^{\prime}\right)\right|^{2} \theta \mathrm{d} x \geqslant C\left|P-P^{\prime}\right|^{2}
$$

for $\left|P-P^{\prime}\right|$ sufficiently small.

Proof. Let $w=u\left(x, P^{\prime}\right)-u(x, P)$ and assume $\bar{H}(P)$ is strictly convex in a neighbourhood of $P$. Then

$$
\begin{aligned}
D_{P} \bar{H}(P)\left(P^{\prime}-P\right) & +C\left|P-P^{\prime}\right|^{2} \\
& \leqslant-\frac{\sigma^{2}}{2} \Delta w+H\left(x, P^{\prime}+D_{x} u\left(x, P^{\prime}\right)\right)-H\left(x, P+D_{x} u(x, P)\right) .
\end{aligned}
$$

Note that

$$
\begin{aligned}
& H\left(x, P^{\prime}+D_{x} u\left(x, P^{\prime}\right)\right)-H\left(x, P+D_{x} u(x, P)\right) \\
& \quad \leqslant D_{p} H\left(x, P+D_{x} u(x, P)\right)\left(P^{\prime}-P+D_{x} w\right)+\Gamma\left|P^{\prime}-P+D_{x} w\right|^{2}
\end{aligned}
$$

for some $\Gamma>0$. Thus

$$
\Gamma \int\left|P^{\prime}+D_{x} u\left(x, P^{\prime}\right)-P-D_{x} u(x, P)\right|^{2} \theta \mathrm{d} x \geqslant C\left|P-P^{\prime}\right|^{2},
$$


since

$$
\left(P^{\prime}-P\right) \int D_{p} H\left(x, P+D_{x} u(x, P)\right) \theta \mathrm{d} x=\left(P^{\prime}-P\right) D_{P} \bar{H}
$$

and

$$
\int-\frac{\sigma^{2}}{2} \Delta w+D_{p} H\left(x, P+D_{x} u(x, P)\right) D_{x} w=0 .
$$

In the case $\sigma=0$, it is possible to prove $L^{\infty}$ estimates on $D_{x x}^{2} u$ on the support of $\theta$ [EG01]. However, this is not the case of $\sigma>0$, at least with estimates independent of $\sigma$. Indeed, if $D_{x x}^{2} u$ were uniformly bounded in $\sigma$ then $u_{\sigma}$ would converge uniformly, through some subsequence as $\sigma \rightarrow 0$, to a function $u$, viscosity solution of

$$
H\left(x, D_{x} u\right)=\bar{H} \text {. }
$$

But then $u$ would be both semiconvex and semiconcave and we know that, in general, $u$ is only semiconcave. However, some regularity exists, as was remarked in section 4, namely one-sided bounds on $D_{x x}^{2} u$ (semiconcavity) that do not depend on $\sigma$.

\section{Explicit formulae and examples}

In this section we discuss several formulae for both $\bar{H}$ and invariant measures. The next proposition shows that, given the solution $u(x, P)$, it is possible to compute the density $\theta$ (under smoothness assumptions), not of the invariant measure but of a time-reversed version. Before stating this proposition, we should observe that, in the case $\sigma=0$, the invariance of the measure $\theta(x, P)$ under the flow is described by the equation

$$
\nabla \cdot(\theta v(x))=0,
$$

i.e. $\theta$ is invariant under the dynamics. Assume further that we can make the change of coordinates

$$
X=x+D_{P} u, \quad p=P+D_{x} u .
$$

In these new coordinates the Hamilton equations (5) are simplified to

$$
\dot{X}=-D_{P} \bar{H}, \quad \dot{P}=0 .
$$

For fixed $P$, the set defined by $p=P+D_{x} u$ is invariant. We turn our attention to invariant measures supported on this set. First observe that since $\dot{X}$ is constant, the measure $\mu$ defined for $A \subset \mathbb{T}^{n}$,

$$
\mu(A)=\int_{A} \mathrm{~d} X
$$

is an invariant probability measure, with respect to the dynamics

$$
\dot{X}=D_{P} \bar{H} \text {. }
$$

The change of coordinates formula yields

$$
\mu(A)=\int_{X^{-1}(A)} \operatorname{det}\left(I+D_{x P}^{2} u\right) \mathrm{d} x .
$$

Therefore, the measure $v$ defined for $B \subset \mathbb{T}^{n}$ by

$$
v(B)=\int_{B} \theta(x, P) \mathrm{d} x,
$$

with $\theta(x, P)=\operatorname{det}\left(I+D_{x P}^{2} u\right)$, is an invariant measure under the dynamics

$$
\dot{x}=D_{p} H\left(P+D_{x} u, x\right) \text {. }
$$


Proposition 5. Assume $u(x, P)$ is a smooth solution of (21). Then

$$
\theta(x, P)=\operatorname{det}\left(I+D_{x P}^{2} u\right)
$$

is a solution of a time-reversed version of (20):

$$
\frac{\sigma^{2}}{2} \Delta \theta+\nabla \cdot(\theta v(x))=0
$$

Proof. Let $v(x, P)=P x+u(x, P)$. Then

$$
-\frac{\sigma^{2}}{2} \Delta v+H\left(x, D_{x} v\right)=\bar{H}(P) .
$$

The claim is that $\theta=\operatorname{det} D_{x P}^{2} v$ solves

$$
-\frac{\sigma^{2}}{2} \Delta \theta+\nabla\left(\theta D_{p} H\left(x, D_{x} v\right)\right)=0
$$

Differentiate (32) with respect to $P$ to get

$$
-\frac{\sigma^{2}}{2} \Delta D_{P} v+D_{p} H D_{x P}^{2} v=D_{P} \bar{H} \text {. }
$$

Note that $D_{p} H D_{x P}^{2} v=\left(D_{x P}^{2} v\right)^{\mathrm{T}} D_{p} H$ and multiply the previous identity by the cofactor matrix cof $D_{x P}^{2} v$ :

$$
-\frac{\sigma^{2}}{2} \operatorname{cof} D_{x P}^{2} v \Delta D_{P} v+\operatorname{det} D_{x P}^{2} v D_{p} H=\operatorname{cof} D_{x P}^{2} v D_{P} \bar{H} .
$$

Observe that cof $D_{x P}^{2} v$ is divergence free [Eva98] and so

$$
\operatorname{cof} D_{x P}^{2} v \Delta D_{P} v=D_{x}\left(\operatorname{det} D_{x P}^{2} v\right) \text {. }
$$

Therefore

$$
-\frac{\sigma^{2}}{2} D_{x}\left(\operatorname{det} D_{x P}^{2} v\right)+\operatorname{det} D_{x P}^{2} v D_{p} H=\operatorname{cof} D_{x P}^{2} v D_{P} \bar{H} .
$$

By applying $\nabla \cdot$ to the previous identity, we have

$$
-\frac{\sigma^{2}}{2} \Delta \theta+\nabla \cdot\left(\theta D_{p} H\right)=0
$$

Now we turn our attention to the special case

$$
H(x, p)=\frac{p^{2}}{2}+V(x),
$$

with $V$ periodic. For this special Hamiltonian, we will present an alternative representation formula for $\bar{H}(P)$ as well as exhibit a (non-periodic) invariant measure. This will follow some ideas of [Hol77].

Suppose $u$ is a periodic viscosity solution of

$$
-\frac{\sigma^{2}}{2} \Delta u+H\left(x, P+D_{x} u\right)=\bar{H}(P) .
$$

Define

$$
\phi=\exp \left(-\frac{P x+u}{\sigma^{2}}\right) .
$$

Then $\phi$ solves

$$
\frac{\sigma^{4}}{2} \Delta \phi+V(x) \phi=\bar{H}(P) \phi .
$$


Thus $\bar{H}$ is an eigenvalue of the operator $\left(\sigma^{4} / 2\right) \Delta \phi+V(x) \phi$. Consider the related operator $L \psi=\mathrm{e}^{P x / \sigma^{2}} \frac{\sigma^{4}}{2} \Delta\left(\mathrm{e}^{-P x / \sigma^{2}} \psi\right)+V(x) \psi=\frac{\sigma^{4}}{2} \Delta \psi-\sigma^{2} P D_{x} \psi+\left(V(x)+\frac{|P|^{2}}{2}\right) \psi$.

Then $\bar{H}$ is also an eigenvalue of $L$ with periodic boundary conditions.

Proposition 6. $\bar{H}$ is the principal eigenvalue of $L$.

Proof. The operator $L$ has a principal eigenvalue $\lambda$ with positive and periodic eigenfunction $\varphi$. Let $u=-\sigma^{2} \log \varphi$. Then $u$ is smooth, periodic and satisfies the Hamilton-Jacobi equation

$$
-\frac{\sigma^{2}}{2} \Delta u+H\left(x, P+D_{x} u\right)=\lambda \text {. }
$$

By uniqueness of $\bar{H}$, we have $\lambda=\bar{H}$.

Next we exhibit an invariant measure for this system, although this is not a probability measure (unless $P=0$ ).

\section{Proposition 7. Let}

$$
\theta=\exp \left(-2 \frac{P x+u}{\sigma^{2}}\right)
$$

Then $\theta$ is an invariant measure.

Proof. It suffices to check that

$$
\frac{\sigma^{2}}{2} \Delta \theta+\nabla\left(\left(P+D_{x} u\right) \theta\right)=0 \text {. }
$$

The next proposition was suggested to me by one of the referees.

Proposition 8. Suppose $V(x)=V(-x)$. Then, for any $P$,

$$
\theta(x)=\exp \left(-\frac{u(x, P)+u(-x, P)}{\sigma^{2}}\right)
$$

is an invariant measure.

Proof. Fix $P$ and write $u(x)=u(x, P)$. Observe that

$$
D_{x} \theta=-\frac{D_{x} u(x)-D_{x} u(-x)}{\sigma^{2}} \theta
$$

and

$$
\Delta \theta=\left(\frac{D_{x} u(x)-D_{x} u(-x)}{\sigma^{2}}\right)^{2} \theta-\frac{\Delta u(x)+\Delta u(-x)}{\sigma^{2}} \theta .
$$

We must show that

$$
\frac{\sigma^{2}}{2} \Delta \theta+\nabla \cdot\left(\left(P+D_{x} u(x)\right) \theta(x)\right)=0 .
$$

Note that

$$
\begin{aligned}
\frac{\sigma^{2}}{2} \Delta \theta= & \theta(x)\left(-\frac{\Delta u(x)+\Delta u(-x)}{2}+\frac{\left(P+D_{x} u(x)\right)^{2}}{2 \sigma^{2}}\right. \\
& \left.+\frac{\left(P+D_{x} u(-x)\right)^{2}}{2 \sigma^{2}}-\frac{\left(P+D_{x} u(x)\right)\left(P+D_{x} u(-x)\right)}{\sigma^{2}}\right)
\end{aligned}
$$


and

$$
\begin{aligned}
& \nabla \cdot\left(\left(P+D_{x} u(x)\right) \theta(x)\right)=\left(P+D_{x} u(x)\right) D_{x} \theta(x)+\Delta u(x) \theta(x) \\
& =\theta(x)\left(\Delta u(x)-\frac{\left|P+D_{x} u(x)\right|^{2}}{\sigma^{2}}+\frac{\left(P+D_{x} u(x)\right)\left(P+D_{x} u(-x)\right)}{\sigma^{2}}\right) .
\end{aligned}
$$

Thus

$$
\begin{aligned}
\frac{\sigma^{2}}{2} \Delta \theta+\nabla \cdot( & \left.\left(P+D_{x} u(x)\right) \theta(x)\right) \\
& =\theta(x)\left(\frac{\Delta u(x)-\Delta u(-x)}{2}-\frac{\left(P+D_{x} u(x)\right)^{2}}{2 \sigma^{2}}+\frac{\left(P+D_{x} u(-x)\right)^{2}}{2 \sigma^{2}}\right) \\
& =\frac{\theta(x)}{\sigma^{2}}(\bar{H}(P)-V(-x)-\bar{H}(P)+V(x))=0
\end{aligned}
$$

since $V(x)=V(-x)$.

\section{Asymptotics}

In this section, we study the asymptotic behaviour of the controlled process $x(t)$. First we will do some formal calculations motivated by the case $\sigma=0$ [EG01, Gom00a, Gom00b]. Define

$$
X=x+D_{P} u \text {. }
$$

Then, by Ito's formula (see [Dur96, p 68]),

$$
\mathrm{d} X=\mathrm{d} x+D_{P x}^{2} u \mathrm{~d} x+\frac{\sigma^{2}}{2} D_{P} \Delta u \mathrm{~d} t .
$$

Thus, since $\mathrm{d} x=-D_{p} H \mathrm{~d} t+\sigma \mathrm{d} w$,

$$
\mathrm{d} X=\left(-D_{p} H\left(I+D_{P x}^{2} u\right)+\frac{\sigma^{2}}{2} D_{P} \Delta u\right) \mathrm{d} t+\sigma\left(1+D_{P x}^{2} u\right) \mathrm{d} w .
$$

Differentiating equation (21) with respect to $P$, we note that $-D_{p} H\left(I+D_{P x}^{2} u\right)+$ $\left(\sigma^{2} / 2\right) D_{P} \Delta u=-D_{P} \bar{H}$ and so

$$
E(X(t)-X(0))=-D_{P} \bar{H} t
$$

since

$$
E \int_{0}^{t} \sigma\left(1+D_{P x}^{2} u\right) \mathrm{d} w=0
$$

Theorem 12. Suppose $\bar{H}$ is differentiable at $P$. Then

$$
\lim _{t \rightarrow \infty} E \frac{x(t)}{t}=-D_{P} \bar{H}
$$

Proof. Let $u$ be a viscosity solution of (21). Let $v^{*}$ be an optimal control such that

$$
u(x, P)=E \int_{0}^{t} L\left(x, v^{*}\right)+P v^{*}+\bar{H}(P)+u(x(t), P) .
$$

Then

$$
u\left(x, P^{\prime}\right) \leqslant E \int_{0}^{t} L\left(x, v^{*}\right)+P^{\prime} v^{*}+\bar{H}\left(P^{\prime}\right)+u\left(x(t), P^{\prime}\right) .
$$


Subtracting these two equations,

$$
C \leqslant E \int_{0}^{t}\left(P^{\prime}-P\right) v^{*}+\bar{H}\left(P^{\prime}\right)-\bar{H}(P) .
$$

Thus

$$
E \int_{0}^{t} v^{*}=-D_{P} \bar{H} t+\mathrm{O}(1) .
$$

Since $\mathrm{d} x=v^{*} \mathrm{~d} t+\sigma \mathrm{d} w$, we have

$$
E \int_{0}^{t} v^{*}=E \int_{0}^{t} \mathrm{~d} x=E x(t)
$$

\section{Convergence as $\sigma \rightarrow 0$}

In this last section we prove that stochastic Mather measures converge to a Mather measure as the diffusion rate $\sigma$ vanishes.

Let $\bar{H}_{\sigma}$ be the unique number for which

$$
-\frac{\sigma^{2}}{2} \Delta u_{\sigma}+H\left(x, D_{x} u_{\sigma}\right)=\bar{H}_{\sigma}
$$

has a periodic viscosity solution $u_{\sigma}$. The bounds on $\bar{H}_{\sigma}$ obtained in section 4 imply that, through some subsequence, $\bar{H}_{\sigma} \rightarrow \bar{H}$ as $\sigma \rightarrow 0$ for some number $\bar{H}$. Since $u_{\sigma}$ is uniformly Lipschitz in $\sigma$, through some subsequence $u_{\sigma} \rightarrow u$ uniformly. Standard stability results on viscosity solutions imply that $u$ is a viscosity solution of

$$
H\left(x, D_{x} u\right)=\bar{H} .
$$

The properties of this limit were studied under certain assumptions of the behaviour of the viscosity solution when $\sigma=0$ by Bessi in [Bes00].

Let $\mu_{\sigma}$ be a stochastic Mather measure associated with (34). Since the support of $\mu_{\sigma}$ is bounded independently of $\sigma$, we can extract a weakly convergent subsequence $\mu_{\sigma} \rightarrow \mu$ and $\int \mathrm{d} \mu=1$. Note that

$$
-\bar{H}_{\sigma}=\int L \mathrm{~d} \mu_{\sigma} \rightarrow \int L \mathrm{~d} \mu=-\bar{H}
$$

Furthermore, for any smooth function $\phi(x)$,

$$
0=\int \frac{\sigma^{2}}{2} \Delta \phi+v D_{x} \phi \mathrm{d} \mu_{\sigma} \rightarrow \int v D_{x} \phi \mathrm{d} \mu .
$$

Thus, $\mu$ satisfies

$$
\int L \mathrm{~d} \mu=-\bar{H}
$$

with the constraints $\int \mathrm{d} \mu=1$ and $\int v D_{x} \phi \mathrm{d} \mu=0$. Thus, $\mu$ is a Mather measure.

\section{Acknowledgments}

I would like to thank the referees for many corrections and improvements. In particular, proposition 8 was suggested to me by one of the referees. 


\section{References}

[Bes00] Bessi U 2000 Aubry-Mather and Hamilton-Jacobi Preprint

[CIPP98] Contreras G, Iturriaga R, Paternain G P and Paternain M 1998 Lagrangian graphs, minimizing measures and Mañé's critical values Geom. Funct. Anal. 8 788-809

[Dur96] Durrett R 1996 Stochastic Calculus (Boca Raton, FL: CRC Press)

[E99] Weinan E 1999 Aubry-Mather theory and periodic solutions of the forced Burgers equation Commun. Pure Appl. Math. 52 811-28

[EG01] Evans L C and Gomes D 2001 Effective Hamiltonians and averaging for Hamiltonian dynamics. I Arch. Ration. Mech. Anal. 157 1-33

[Eva98] Evans L C 1998 Partial Differential Equations (Providence, RI: American Mathematical Society)

[Fat97a] Fathi A 1997 Solutions KAM faibles conjuguées et barrières de Peierls C. R. Acad. Sci. Paris Sér. I Math. 325 649-52

[Fat97b] Fathi A 1997 Théorème KAM faible et théorie de Mather sur les systèmes lagrangiens $C$. R. Acad. Sci. Paris Sér. I Math. 324 1043-6

[Fat98a] Fathi A 1998 Orbite hétéroclines et ensemble de Peierls C. R. Acad. Sci. Paris Sér. I Math. 326 1213-6

[Fat98b] Fathi A 1998 Sur la convergence du semi-groupe de Lax-Oleinik C. R. Acad. Sci. Paris Sér. I Math. 327 $267-70$

[Fle89] Fleming W H 1989 Generalized solutions and convex duality in optimal control Partial Differential Equations and the Calculus of Variations vol I (Boston, MA: Birkhäuser) pp 461-71

[FS93] Fleming W H and Mete Soner H 1993 Controlled Markov Processes and Viscosity Solutions (New York: Springer)

[FV88] Fleming W H and Vermes D 1988 Generalized solutions in the optimal control of diffusions Stochastic Differential Systems, Stochastic Control Theory and Applications (Minneapolis, MN, 1986) (New York: Springer) pp 119-27

[FV89] Fleming W H and Vermes D 1989 Convex duality approach to the optimal control of diffusions SIAM J. Control Optim. 27 1136-55

[Gom00a] Gomes D 2000 Viscosity solutions of Hamilton-Jacobi equations, and asymptotics for Hamiltonian systems Preprint

[Gom00b] Gomes D A 2000 Hamilton-Jacobi equations, viscosity solutions and asymptotics of Hamiltonian systems PhD Thesis University of California at Berkeley

[Hol77] Holland C J 1977 A new energy characterization of the smallest eigenvalue of the Schrödinger equation Commun. Pure Appl. Math. 30 755-65

[Kry87] Krylov N V 1987 Nonlinear Elliptic and Parabolic Equations of the Second Order (translated from the Russian by P L Buzytskiü)

[LV80] Lewis R M and Vinter R B 1980 Relaxation of optimal control problems to equivalent convex programs J. Math. Anal. Appl. 74 475-93

[Mat89] Mather J N 1989 Minimal action measures for positive-definite Lagrangian systems IXth Int. Congr. on Mathematical Physics (Swansea, 1988) (Bristol: Hilger) pp 466-8

[Mat91] Mather J N 1991 Action minimizing invariant measures for positive definite Lagrangian systems Math. Z. 207 169-207

[Mat01] Mather J N 2001 Personal communication

[Mn92] Mañé R 1992 On the minimizing measures of Lagrangian dynamical systems Nonlinearity 5 623-38

[Mn96] Mañé R 1996 Generic properties and problems of minimizing measures of Lagrangian systems Nonlinearity $9273-310$

[PW84] Protter M H and Weinberger HF 1984 Maximum Principles in Differential Equations (New York: Springer) (corrected reprint of the 1967 original)

[Roc66] Rockafellar R T 1966 Extension of Fenchel's duality theorem for convex functions Duke Math. J. 33 81-9

[VL78a] Vinter R B and Lewis R M 1978 The equivalence of strong and weak formulations for certain problems in optimal control SIAM J. Control Optim. 16 546-70

[VL78b] Vinter R B and Lewis R M 1978 A necessary and sufficient condition for optimality of dynamic programming type, making no a priori assumptions on the controls SIAM J. Control Optim. 16 571-83 